University of Wollongong

Research Online

Faculty of Social Sciences - Papers (Archive) Faculty of Arts, Social Sciences \& Humanities

2013

Child, family and environmental correlates of children's motor skill proficiency

Lisa M. Barnett

Deakin University

Trina Hinkley

Deakin University, thinkley@uow.edu.au

Anthony D. Okely

University of Wollongong, tokely@uow.edu.au

Jo Salmon

Deakin University

Follow this and additional works at: https://ro.uow.edu.au/sspapers

Part of the Education Commons, and the Social and Behavioral Sciences Commons

Research Online is the open access institutional repository for the University of Wollongong. For further information contact the UOW Library: research-pubs@uow.edu.au 


\title{
Child, family and environmental correlates of children's motor skill proficiency
}

\author{
Abstract \\ To identify factors associated with children's motor skills. Cross-sectional. Australian preschool-aged \\ children were recruited in 2009 as part of a larger study. Parent proxy-report of child factors (age, sex, \\ parent perception of child skill, participation in unstructured and structured activity), self-report of parent \\ factors (confidence in their own skills to support child's activity, parent-child physical activity interaction, \\ parent physical activity) and perceived environmental factors (play space visits, equipment at home) were \\ collected. Moderate to vigorous physical activity (MVPA) (ActiGraph GT1M accelerometer) and motor \\ skills (Test of Gross Motor Development-2) were also assessed. After age adjustment, variables were \\ checked for association with raw object control and locomotor scores. Variables with associations of $p<$ \\ 0.20 were entered into two multiple regression models with locomotor/object control as respective \\ outcome variables. Motor skills were assessed for 76 children (42 female), mean [SD] age-4.1 [0.68]: 71 \\ completed parent proxy-report and 53 had valid MVPA data. Child age, swimming lessons, and home \\ equipment were positively associated explaining $20 \%$ of locomotor skill variance, but only age was \\ significant $(\beta=0.36, p=0.002)$. Child age and sex, unstructured activity participation, MVPA\%, parent \\ confidence, home equipment (all positively associated), and dance participation (inversely associated) \\ explained $32 \%$ object control variance. But only age $(\beta=0.67, p<0.0001)$, MVPA $\%(\beta=0.37, p=0.038)$ \\ and no dance $(\beta=-0.34, p=0.028)$ were significant. Motor skill correlates differ according to skill \\ category and are context specific with child level correlates appearing more important.
}

\section{Keywords}

era2015, proficiency, children, skill, correlates, environmental, family, child, motor

\section{Disciplines}

Education | Social and Behavioral Sciences

\section{Publication Details}

Barnett, L. M., Hinkley, T., Okely, A. D. \& Salmon, J. (2013). Child, family and environmental correlates of children's motor skill proficiency. Journal of Science and Medicine in Sport, 16 (4), 332-336. 
1 Child, family and environmental correlates of children's motor skill proficiency 


\section{Abstract}

2 Objectives: To identify factors associated with children's motor skills. Design: Cross-sectional.

3 Methods: Australian preschool-aged children were recruited in 2009 as part of a larger study. Parent

4 proxy-report of child factors (age, sex, parent perception of child skill, participation in unstructured

5 and structured activity), self-report of parent factors (confidence in their own skills to support child's

6 activity, parent-child physical activity interaction, parent physical activity) and perceived

7 environmental factors (play space visits, equipment at home) were collected. Moderate to Vigorous

8 Physical activity (MVPA) (Actigraph GT1M accelerometer) and motor skills (Test of Gross Motor

9 Development-2) also assessed. After age adjustment, variables were checked for association with raw

10 object control and locomotor scores. Variables with associations of $\mathrm{p}<.20$ were entered into two

11 multiple regression models with locomotor/object control as respective outcome variables. Results:

12 Motor skills were assessed for 76 children (42 female), mean [SD] age $=4.1[0.68]$; 71 completed

13 parent proxy-report and 53 had valid MVPA data. Child age, swimming lessons, and home equipment

14 were positively associated explaining $20 \%$ of locomotor skill variance; but only age was significant ( $\beta$

$15=0.36, \mathrm{p}=0.002$ ). Child age and sex, unstructured activity participation, MVPA\%, parent confidence,

16 home equipment (all positively associated), and dance participation (inversely associated), explained

$1732 \%$ object control variance. But only age $(\beta=0.67, \mathrm{p}<0.0001)$, MVPA\% $(\beta=0.37, \mathrm{p}=0.038)$ and

18 no dance $(\beta=-0.34, p=0.028)$, were significant. Conclusion: Motor skill correlates differ according

19 to skill category and are context specific with child level correlates appearing more important. 


\section{Introduction}

2 Motor skill competence (e.g. the ability to throw, kick and jump proficiently) is linked to physical

3 activity in both children and adolescents ${ }^{1}$. Understanding more about motor skill competence in

4 young children (three to five years old) may be particularly important, as only $3 \%$ to $5 \%$ of their time

5 is spent in moderate to vigorous activity ${ }^{2-4}$, and this early period has been identified as crucial in the

6 establishment of physical activity behaviours and motor skills ${ }^{5,6}$.

7 Ecological systems theory conceptualises child behaviour as stemming from interactions between the

8 various influences that impact on a child ${ }^{7}$. The contextual influences as applied to physical activity

9 behaviour, are characteristics associated with the child, the family, and the environment ${ }^{8}$. Whilst

contextual influences on children's physical activity have been explored, little has been undertaken with respect to motor skill proficiency.

Given the association between physical activity and motor competence, it is possible that the contextual factors that are associated with motor skill proficiency may be similar to those for physical activity. For instance, participation in outdoor unstructured play was associated with pre-schoolers’ physical activity ${ }^{9}$ and may also be linked to children's motor skill. In another study, parent perception of their child's motor skill was associated with their child's physical activity level ${ }^{10}$.

Family socio-cultural factors (such as parental physical activity and interaction) are associated with physical activity in pre-school aged children ${ }^{9}$ and there is some evidence they may also influence motor skill. For instance, Cools et al. recently found in a large sample $(n=846)$, that fathers' physical activity level was positively associated with fundamental motor skills in preschool boys ${ }^{11}$.

Physical environmental influences such as time and frequency in play spaces outside the home are associated with pre-schoolers' physical activity ${ }^{9}$, but there is little information as to whether these factors are associated with motor skill in this age group. Possessing toys at home might be 
1 hypothesised to positively impact on motor skill because of the increased opportunity for

2 children to practice certain skills (e.g. ball for practising the throw). Conversely, children who

3 have better skills may be likely to request new sports or physical activity related equipment.

4 In fact, the Cools et al. study did find that the frequency which parents bought new equipment

5 for their child was positively associated with their motor skills ${ }^{11}$.

6 The purpose of the current study was to determine the contextual correlates of children's

7 motor skills. In particular, which child, family and environmental factors were related to

8 locomotor and object control skills in preschool aged children.

\section{Methods}

Children were recruited as part of the Healthy Active Preschool Years (HAPPY) study. All metropolitan councils $(n=29)$ were divided into quintiles, based on the 2001 Socio-economic Indices for Areas index of advantage and disadvantage ${ }^{12}$. Two local government areas (LGAs) from each of

13 the lowest, middle and highest quintiles were randomly selected. Within each of these LGA's, 14 preschools/childcare centres were recruited from a randomly ordered list of centres. Recruitment and data collection occurred during 2008/09. In total, 156 childcare centres and 137 preschools were approached. Following centre approval, all parents of children three years or older were invited. Parent consent rate was 11\% $(n=1032 / 9825)$. A subset of children recruited in $2009(11 \%, n=76 / 707$ from 12 centres) were asked to consent to their children's motor skills assessment, in addition to other measures conducted as part of HAPPY described below. Ethics approval was received from Deakin University and Department of Education and Early Childhood Development.

To objectively assess child physical activity, children were fitted with an ActiGraph GT1M accelerometer and instructed that the accelerometer was to be worn on the right hip, from waking for the entire day, removed only for sleeping and aquatic activities. Data were collected in 15-second epochs to maximise opportunities to more accurately capture the sporadic nature of young children's physical activity ${ }^{4,13}$. Children wore accelerometers for an eight-day period. Each monitor was 
1 initialised on the first day to commence recording at $9 \mathrm{am}$, and data management adjusted for actual

2 wear-time. Participants' data were included if they had a minimum of three week and one weekend

3 days of data, with data for at least $50 \%$ of their usual wake time on each day. Spearman-Brown

4 analysis ${ }^{14}$ on the larger HAPPY sample indicated that up to 2.5 weekdays, and 0.7-1.4 weekend days,

5 were required to achieve generally accepted reliability of $0.8{ }^{15}$. Percentage of time spent in moderate

6 and vigorous physical activity (MVPA) was determined for each day individually, and across the

7 entire wear period, using age specific cut-points ${ }^{16}$. After checking for normality, child percentage of

8 time in MVPA was log transformed before analysis.

9 Parents completed a survey in which they reported their child's date of birth and sex, their own sex, country of birth, highest level of education, and employment, and whether they or their partner (where relevant) had a health care or pension card (an indication of low income status). The survey included child, family and environmental factors hypothesised to be associated with motor skills. Test-retest reliability (14-50 days, mean 24) was conducted on a separate parent sample $(n=47)$ on these survey

14 factors. All categorical variables used in the current study had a kappa $>0.4$ and \% agreement $>0.6$, and all continuous variables had an ICC $>0.50$.

Child skill (as perceived by the parent) was assessed by asking whether they agreed or disagreed that their child doesn't have good enough motor skills to participate in physical activity. Response options were on a five point scale from Strongly Disagree (1) to Agree (5). Structured activity participation was determined by whether their child participated (yes, no) in swimming, dance and kindy-gym in the last month. Unstructured activities referred to how often their child usually: rode a bike /scooter for fun, played in the backyard, played on a trampoline/swing/other equipment, used toys and equipment such as bats and balls in his/her play, and/or swam in a pool. The six point scales for each item were summed with a possible score ranging from 5 to 30 .

Parents were asked if they felt confident they had the skills to support their child to be active. Options were collapsed into 'not confident' and 'confident'. Parental interaction in child's physical activity was assessed by two items: How often are you and/or your partner physically active with your child? 
1 Response options were on a six point scale ranging from never (1) to daily (6). The two items were

2 summed (except for four parents without partners who were excluded) to create a score ranging from 2

3 to 12. Respondent parents were also asked to report their own moderate- and vigorous-intensity

4 physical activity (engaged in for at least 10 minutes at a time) in a typical week; item taken from the

5 Active Australia Survey ${ }^{17}$. Parent time (mins/week) in moderate, and also vigorous, activity were each

$6 \quad \log$ transformed before analysis.

7 Frequency of visits to a play space was assessed by asking how often your preschool child would

8 usually go to: local playground, playground in another area, parks/ovals with no play equipment,

9 sports venue, specialist outdoor activity centre, indoor play centre. Response options for the six items were on a seven point scale ranging from never (1) to five or more times a week (7) and were summed to range from 6 to 42. Parents were also asked to specify toys/equipment at home suitable for physical activity. Equipment items considered to be related to motor skills were: balls, basketball ring, bats racquets or golf clubs, bowls, climbing equipment/trees suitable for climbing, frisbee, pool or beach toys, roller blades or skates, scooter, skateboard, skipping rope, slide, soft balls for indoor play, swings, swimming/wading pool, table tennis table bats and balls, trampoline and volleyball/badminton net, and/or a tricycle/bicycle. Availability of items was summed into a composite with possible scores ranging from 0 to 18.

The Test of Gross Motor Skill Development $-2^{\text {nd }}$ Edition (TGMD-2) was used to assess six locomotor skills (running, galloping, hopping, leaping, horizontal jumping, sliding) and six object control skills (striking a stationary ball, stationary dribble, kicking, catching, overhand throwing, and underhand rolling) ${ }^{18}$. The TGMD-2 is a process oriented measure, assessing the components of each skill rather than the outcome or product of the skill execution.

Children were given a demonstration of the proficient technique before being asked to perform the skill twice. Encouragement was given but no verbal feedback provided on skill performance. Each attempt was scored with each component of the skill receiving a ' 1 ' if correctly executed or a ' 0 ' if not. Each skill had a number of components (ranging from 3 to 5) that needed to be demonstrated for 
1 the skill to be performed proficiently. Scores of the two trials were summed to obtain a raw score for

2 each skill. The six locomotor skill scores for each child were then summed for the locomotor subset

3 and the six object control scores for each child were summed for the object control subset.

4 All children were assessed in the preschool/childcare setting using live observation by observers

5 trained for that purpose. The training of four observers (12 hours) was conducted in 2009 by an expert trainer. Accuracy was assessed by comparing observer scores (0-10 depending on skill) to the expert trainers' ratings of videotapes of children performing each skill. Training was completed when observer scores were no more than one point difference to the expert trainers' ratings. In the field, inter-rater reliability was assessed opportunistically with each observer matched with each other observer at least once. Interrater reliability for raw totals for the object control and locomotor subsets were assessed using an intraclass correlation (ICC) with a one way model for absolute agreement for single measures. Inter-rater reliability was assessed on 16 children (21\% of sample). Inter-rater reliability was strong for both the locomotor raw subtotal scores (ICC $=.92,95 \%$ CI .80 to .97) and for the object control raw subtotal (ICC $=.90,95 \%$ CI .74 to .96 ).

SPSS version 17.0 was used for analyses. Variables were firstly entered one at a time into respective regression models to check for associations with a) locomotor skills (raw subtest score) or b) object control skills (raw subtest score). As age is a known determinant of motor skills, associations were adjusted for age in days. Those variables with associations of $\mathrm{p}<0.20$ with object control or locomotor skill (after age adjustment) were entered into two standard multiple regression models. This less stringent significance level $(\mathrm{p}<0.20$ ) was chosen for variable inclusion in the model/s, because variables may contribute to a regression model in unexpected ways due to the potentially complex interrationships among the variables ${ }^{19}$. There was no evidence of multicollinearity for either model, with the tolerance for each predictor variable in both models over 0.6; indicating that each variable could not be well accounted for by other variables. Also, the variance inflation factors were all under 10 , indicating the standard errors for the coefficient were unlikely to be inflated ${ }^{20}$. The determined significance level for variables in the final models was $\mathrm{p}<0.05$. 
2 Slightly more than half ( $n=42 ; 55 \%$ ) of the 76 children were girls. Age ranged from three to six years

3 (mean 4.1, $S D=0.68$ ). All had complete motor skill assessments, 71 parents completed the proxy-

4 report survey, and 53 children met the inclusion criteria for valid physical activity. The sample of

5 respondent parents were almost all female $(n=68,95.8 \%)$ with 66.2\% Australian born and the

6 remainder born in 15 other countries. Most were working full- or part-time (69.0\%) with 28.2\%

7 engaged full-time in home duties, (87.3\%) had a University degree or Year 12/Diploma/trade

8 certificate (8.5\%), and five (7.0\%) families had a health care card. See Table 1.

INSERT TABLE 1

Nearly all parents disagreed with the statement that their child doesn't have good enough motor skills

to participate in physical activity, with only one agreeing and a further three neither agreeing nor disagreeing; therefore this variable was not included in further analyses. After age adjustment, the only child factor associated with locomotor skill was participation in swimming lessons. Child factors associated with object control skill, included sex, participation in unstructured physical activity, MVPA (\%) and not participating in dance classes. Of the family factors, none were associated with locomotor skills and parents' confidence in their own skills was associated with the child's object control skills. In terms of environmental factors, the number of pieces of equipment at home was associated with both locomotor and object control skills (Tables 2 and 3).

In the final locomotor model, child age, participation in swimming lessons, and equipment explained $20 \%$ of locomotor skill variance; but participation in swimming lessons and equipment did not reach significance (Table 2). In the final object control model, child age and sex, unstructured physical activity, MVPA (\%), not participating in dance classes, parent confidence of own skill, and number of of toys/equipment in the home explained 32\% of object control variance; although only child age, MVPA (\%) and not participating in dance classes remained significant (Table 3). 
INSERT TABLE 2, and 3

\section{Discussion}

4 This is one of few studies to use an ecological systems framework to explore factors associated with

5 children's motor skills ${ }^{11}$. Our results are important because understanding which factors are

6 associated with motor skills may be key in helping young children develop motor skill competency. A

7 range of factors at the child, family and environment levels were initially associated with motor skill,

8 supporting the use of the ecological framework in understanding correlates of motor skill. However,

9 there were some differences between the factors associated with locomotor and object control skills. In the final models, only factors at the child level were associated with either skill type, indicating that whilst factors supporting the development of motor skill competency appear to be multidimensional,

12 factors specific to the child are most important.

Child age, child MVPA and not participating in dance classes were all statistically significant in the final object control model. Our finding that child MVPA was positively associated with object control skill and not locomotor skill support those in an adolescent sample in which MVPA explained more variance in object control skills than locomotor skills (12\% compared to $2 \%)^{21}$. Our finding is also consistent with Cliff et al. who reported that object control skills were related to time in MVPA in preschool boys $(\mathrm{n}=25)^{22}$.

The inverse association with participating in dance classes and object control skills is interesting. This may signify that those children who participate in dance are spending less time developing their object control skills due to interest, time or other factors. Another explanation is that more girls participated in dance and the girls had poorer object control skills, in which case dance participation could be seen as a proxy for being female; similarly the converse is also relevant. Studies in older children have found sex differences in motor skill type with boys generally more proficient in object control skills than girls ${ }^{23-25}$. To test this explanation, further investigation of the data was undertaken. Of the 26 children who participated in dance around a quarter were boys $(n=7)$. When we split the file by sex and ran a regression with structured dance participation as a predictor and object control skill as an 
outcome, structured dance participation was significant and negatively associated for girls only. This

2 indicates that the girls who dance, were less likely to have good object control skills.

3 Child age was the only factor significantly positively associated with locomotor skill in the final

4 model although participation in swimming classes was close to significant $(\mathrm{p}=.05)$. A study in older

5 children also found participation in structured sporting activities was associated with higher motor

6 skill proficiency ${ }^{26}$. However, we found that participation in dance and kindy-gym classes had no

7 association with locomotor skills. It is unclear why swimming appeared to have more association with

8 locomotor skills than dance and kindy-gym, as it would appear that all three activities involve

9 locomotor skills; for instance 'leaping' in dance or 'jumping' in kindy-gym. Perhaps swimming may require more whole body involvement (i.e. all four limbs). Our finding that the associations between structured activity participation and motor skill proficiency differ according to the nature of the activity, indicate that it is important to be able to specify the actual activity context to be able to detect associations with motor skill type.

Having skill-related equipment present in the home was positively associated with locomotor and object control skill after age adjustment. This suggests that having a supportive environment in terms of toys and equipment may help develop motor skill competence, or that children with better locomotor and object control skills are provided with more equipment. The Cools et al. study supports this finding, as the frequency which parents bought new equipment for their child was associated with motor skill ${ }^{11}$.

Our finding that child participation in unstructured activity was only positively associated with object control skill after adjusting for age, but not in the final model, indicates that time spent in MVPA and context specific activity were more important to object control skills, than general physical activities. Parental confidence in their skills to support their child to be active was also only associated with object control skill, after adjusting for age. It is unclear why parent confidence would only be associated with object control skills but not locomotor skills. Perhaps object control skills rely more heavily on parent demonstration and instruction than locomotor skills. Both object control and 
1 locomotor skills can be viewed as phylogenetic and therefore needing environmental support for their

2 development ${ }^{27}$, but it is unclear how much support is needed. It is feasible that object control skills

3 require more direct support from parents at this young age, for example, young children need parents

4 to throw them a ball to practice catching and to collect it once it's been kicked or thrown. Children in

5 our sample had not yet commenced any sort of school physical education and whilst some participated

6 in structured activities, these activities do not generally include object control skill instruction. It is

7 also possible that parents may also have considered other skills (e.g. confidence in their ability to be

8 active with their child) in relation to this question.

9 The main limitation of this study is the small sample size. It is possible that with a larger sample we may have been better able to tease out the contribution of family and environmental factors. As a child

11 ages family and environmental factors may become of more importance. Stodden et al. suggests that

12 as a child ages the relationship between individual, family and environmental constraints will

13 compound and relate more strongly over time to movement skill competence and physical activity

14 behaviour ${ }^{28}$.

15 Also, the sample is not likely to be representative given the high level of parents' education; although the sample was ethnically diverse. Nevertheless, the strengths are the objective measurement of physical activity and motor skill, and the use of a survey at the child, family and environment level to investigate this little explored topic.

\section{Conclusion}

This study has shown that the association between motor skill and its correlates is complex and differs according to skill type. Whilst there is some evidence that motor skill in pre-school aged children is multidimensional, being associated with factors at the child, family and environmental level, in the final models only factors at the child level were associated with motor skills. 
- What activity a child does, not how much physical activity, is important to locomotor skill development

- What activity a child does and how much physical activity is important to object control skill

4 development

- Interventions aiming to increase a young child's skill level should focus on child level individual factors first.

\section{Acknowledgements}

8 This study was funded by Deakin University. Thank you to the research assistants, and children and 9 parents who participated. 
21 Lubans DR, Morgan PJ, Cliff DP, Barnett LM, Okely AD. Review of the benefits 3 associated with fundamental movement skill competency in youth. Sports Medicine. 2010; 4 40: 1019-35.

52 Jackson DM, Reilly JJ, Kelly LA, Montgomery C, Grant S, Paton JY. Objectively 6 measured physical activity in a representative sample of 3- to 4- year-old children. Obesity $7 \quad$ Research. 2003; 11: 420-25.

83 Fisher A, Reilly JJ, Kelly LA, Montgomery C, Williamson A, Paton J, et al. 9 Fundamental movement skills and habitual physical activity in young children. Medicine and 10 Science in Sports and Exercise. 2005; 37: 684-88.

114 Montgomery C, Reilly J, Jackson D, Kelly L, Slater C, Paton J, et al. Relation 12 between physical activity and energy expenditure in a representative sample of young 13 children. American Journal of Clinical Nutrition. 2004; 80: 591-96.

145 Gallahue DL, Ozmun JC. Understanding motor development: infants, children, 15 adolescents, adults. 4th edn: Boston, Mass.McGraw-Hill, 1998.

166 Birch LL, Fisher JO. Development of eating behaviors among children and 17 adolescents. Pediatrics. 1998; 101: 539.

7 Bronfenbrenner U, Morris P. The ecology of human developmental processes. In: Damon W, Eisenberg N (eds.). The handbook of child psychology 3rd edn John Wiley \&

20 Sons: New York 1988; 993-1027.

218 Davison KK, Birch LL. Childhood overweight: a contextual model and 22 recommendations for future research. Obesity Reviews. 2001; 2: 159-71.

239 Hinkley T, Crawford D, Salmon J, Okely AD, Hesketh K. Preschool children and 24 physical activity: a review of correlates. American Journal of Preventive Medicine. 2008; 34: 25 435-41.

2610 Bois JE, Sarrazin PG, Brustad RJ, Trouilloud DO, Cury F. Elementary 27 schoolchildren's perceived competence and physical activity involvement: the influence of 28 parents' role modelling behaviours and perceptions of their child's competence. Psychology of 29 Sport and Exercise. 2005; 6: 381-97.

3011 Cools W, Martelaer K, D., Samaey C, Andries C. Fundamental movement skill 31 performance of preschool children in relation to family context. Journal of Sports Sciences 32 2011; 29: 649 - 60.

3312 Australian Bureau of Statistics. Census of Population and Housing: Socio-Economic 34 Indexes for Areas (SEIFA) In: Australian Bureau of Statistics (ed.): Canberra. 2001. 
113 Cliff DP, Reilly JJ, Okely AD. Methodological considerations in using accelerometers to 2 assess habitual physical activity in children aged 0-5 years. . Journal of Science and Medicine in 3 Sport 2009; 12 557-67.

414 Stanley JC. Reliability. In: Thorndike RL (ed.). Educational Measurement. American

5 Council of Education, : Washington, DC, 1971; 395.

615 Trost SG, Pate R, Freedson PS, Sallis JF, W.C. T. Using objective physical activity 7 measures with youth: how many days of monitoring are needed? . Medicine and Science in 8 Sports and Exercise. 2000; 32 426-31.

916 Sirard JR, Trost SG, Pfeiffer KA, Dowda M, Pate RR. Calibration and evaluation of 10 an objective measure of physical activity in preschool children. Journal of Physical Activity 11 and Health. 2005; 2.

17 Brown WJ, Trost SG, Bauman A, Mummery K, Owen N. Test-retest reliability of four physical activity measures used in population surveys. Journal of Science And Medicine In Sport. 2004; 7 205-15.

18 Ulrich DA. Test of Gross Motor Development (2nd ed). Austin, TX 2000.

19 Altman DG. Practical statistics for medical research: Chapman and Hall 1991.

20 UCLA: Academic Technology Services SCG. Regression with SPSS, Chapter 2 Regression Diagnostics. Introduction to SAS SPSS Web Books, . Actual and Perceived Skill Proficiency and Physical Activity. Medicine and Science in Sports and Exercise. 2011; 43: 898-904.

22 Cliff DP, Okely AD, Smith LM, McKeen K. Relationships between fundamental movement skills and objectively measured physical activity in preschool children. Pediatric Exercise Science. 2009; 21: 436-49.

23 Hume C, Okely AD, Bagley S, Telford A, Booth M, Crawford D, et al. Does weight status influence associations between children's fundamental movement skills and physical activity? Research Quarterly for Exercise and Sport. 2008; 79: 158-65.

24 Raudsepp L, Paasuke M. Gender differences in fundamental movement patterns, movement performances, and strength measurements in prepubertal children. Pediatric Exercise Science. 1995; 7: 294-304.

25 van Beurden E, Zask A, Barnett LM, Dietrich UC. Fundamental movement skills How do primary school children perform? The 'Move It Groove It' program in rural Australia. Journal of Science and Medicine in Sport. 2002; 5: 244-52. 
$127 \quad$ Clark JE. On the problem of motor skill development: motor skills do not develop 2 miraculously from one day to the next. They must be taught and practiced. Journal of 3 Physical Education Recreation and Dance. 2007; 78 39-44.

428 Stodden DF, Goodway JD, Langendorfer SJ, Roberton MA, Rudisall ME, Garcia C, et 5 al. A developmental perspective on the role of motor skill competence in physical activity:

6 An emergent relationship. Quest. 2008; 60: 290-306.

7

8 